\title{
Fcc and bec structured ferromagnetic films with five to twenty spin layers explained by second order perturbed Heisenberg Hamiltonian
}

\author{
P. Samarasekara* and T.H.Y.I.K. de Silva \\ Department of Physics, University of Peradeniya, Peradeniya, Sri Lanka
}

\begin{abstract}
Second order perturbed 2-D Heisenberg Hamiltonian model was employed to explain the magnetic properties of fcc and bcc structured ferromagnetic thin films with five to twenty spin layers. Magnetic energy versus azimuthal angle of spin was plotted to find the angle corresponding to maximum energy and minimum energy. Hence magnetic easy and hard directions were determined. The magnetic easy axis gradually rotates from out of plane (perpendicular) to in plane direction as the number of spin layers is increased. This implies that the films indicates a preferred in plane easy axis orientation at higher thicknesses. The energy required to rotate spin from hard to easy (or vice versa) directions gradually increases with number of spin layers. Our results agree with the experimental data obtained for ferromagnetic thin films.
\end{abstract}

Keywords: Heisenberg Hamiltonian, thin films, magnetic easy axis, ferromagnetic.

\section{INTRODUCTION}

Ferromagnetic thin films are applied in magnetic memory devices, some miniature devices and microwave devices. Compared with the bulk magnetic materials, the same magnetic properties can be obtained using an easy axis oriented ferromagnetic thin film with a much less volume in miniature devices. As a matter of fact, the advantage of ferromagnetic thin films is attributed to the size of the sample. Stress induced anisotropy plays a major role in magnetic thin films. Both classical and quantum models of spins are applied to explain the behavior of magnetic materials. EuTe films with surface elastic stresses have been theoretically studied by means of Heisenberg Hamiltonian (Radomska and Balcerzak, 2003). Magnetostriction of dc magnetron sputtered FeTaN thin films has been investigated using the theory of De Vries (Cates and Alexander, 1994). Ferromagnetic multilayers of $\mathrm{Ni}$ on $\mathrm{Cu}$ have been theoretically investigated using the Korringa-Kohn-Rostoker Green's function method (Ernst et al., 2000). Electric and magnetic properties of multiferroic thin films have been described by modified Heisenberg and transverse Ising model using Green's function technique (Kovachev and Wesselinowa, 2009). The quasistatic magnetic hysteresis of ferromagnetic thin films grown on a vicinal substrate has been theoretically investigated by Monte Carlo simulations (Zhao et al., 2002). Structural and magnetic properties of two dimensional $\mathrm{Fe}-\mathrm{Co}$ orders alloys deposited on tungsten substrates have been studied using first principles band structure theory (Spisak and Hafner, 2005).

The same Heisenberg Hamiltonian model with spin exchange interaction, magnetic dipole interaction, applied magnetic field, second and fourth order magnetic anisotropy terms have been studied previously (Hucht and Usadel, 1997; Hucht and Usadel,1999, Usadel and Hucht, 2002). The Heisenberg Hamiltonian model was modified by including stress induced anisotropy and demagnetization factor by us. Domain structure and Magnetization reversal in thin magnetic films have been theoretically investigated (Nowak, 1995). In-plane dipole coupling anisotropy of a square ferromagnetic Heisenberg monolayer has been explained using Heisenberg Hamiltonian (Dantziger et al., 2002). Effect of the interfacial coupling on the magnetic ordering in ferro-antiferromagntic bilayers has been studied using the Heisenberg Hamiltonian (Tsai et al., 2003).

This modified Heisenberg Hamiltonian was employed to investigate the third order perturbed energy of ferromagnetic thick films (Samarasekara, 2008), second order perturbed energy of ferrite thin films (Samarasekara et al., 
2009), third order perturbed energy of ferromagnetic thin films (Samarasekara and Mendoza, 2010), second order perturbed energy of thick ferrite films (Samarasekara 2010), third order perturbed energy of thick ferrite films (Samarasekara, 2011) and third order perturbed energy of thin ferrite films ((Samarasekara and
Mendoza, 2011). In addition, spin reorientation of ferromagnetic films (Samarasekara and Gunawardhane 2011), nickel ferrite films (Samarasekara and Saparamadu, 2012) and barium ferrite films (Samarasekara and Saparamadu, 2013) were investigated using Heisenberg Hamiltonian.

\section{MODEL}

The modified classical Heisenberg Hamiltonian of ferromagnetic films is given as following.

$$
\begin{aligned}
H=-\frac{J}{2} \sum_{m, n} \vec{S}_{m} \cdot \vec{S}_{n}+\frac{\omega}{2} \sum_{m \neq n}\left(\frac{\vec{S}_{m} \cdot \vec{S}_{n}}{r_{m n}{ }^{3}}-\frac{3\left(\vec{S}_{m} \cdot \vec{r}_{m n}\right)\left(\vec{r}_{m n} \vec{S}_{n}\right)}{r_{m n}{ }^{5}}\right)-\sum_{m} D_{\lambda_{m}}{ }^{(2)}\left(S_{m}{ }^{z}\right)^{2}-\sum_{m} D_{\lambda_{m}}{ }^{(4)}\left(S_{m}{ }^{z}\right)^{4} \\
\quad-\sum_{m, n}\left[\vec{H}-\left(N_{d} \vec{S}_{n} / \mu_{0}\right)\right] \cdot \vec{S}_{m}-\sum_{m} K_{s} \operatorname{Sin} 2 \theta_{m}
\end{aligned}
$$

Here, $\vec{S}_{m}$ and $\vec{S}_{n}$ are two spins. Above equation can be deduced to following form.

$$
\begin{aligned}
E(\theta)=- & \frac{1}{2} \sum_{m, n=1}^{N}\left[\left(J Z_{|m-n|}-\frac{\omega}{4} \Phi_{|m-n|}\right) \cos \left(\theta_{m}-\theta_{n}\right)-\frac{3 \omega}{4} \Phi_{|m-n|} \cos \left(\theta_{m}+\theta_{n}\right)\right] \\
& -\sum_{m=1}^{N}\left(D_{m}{ }^{(2)} \cos ^{2} \theta_{m}+D_{m}{ }^{(4)} \cos ^{4} \theta_{m}+H_{\text {in }} \sin \theta_{m}+H_{\text {out }} \cos \theta_{m}\right) \\
& +\sum_{m, n=1}^{N} \frac{N_{d}}{\mu_{0}} \cos \left(\theta_{m}-\theta_{n}\right)-K_{s} \sum_{m=1}^{N} \sin 2 \theta_{m}
\end{aligned}
$$

Where $J, Z_{|m-n|}, \omega, \Phi_{|m-n|}, \theta, D_{m}{ }^{(2)}, D_{m}{ }^{(4)}, H_{i n}, H_{\text {out }}, N_{d}, K_{s}, \quad m, n$ and $N$ are spin exchange interaction, number of nearest spin neighbors, strength of long range dipole interaction, constants for partial summation of dipole interaction, azimuthal angle of spin, second and fourth order anisotropy constants, in plane and out of plane applied magnetic fields, demagnetization factor, stress induced anisotropy constant, spin plane indices and total number of layers in film, respectively.

In terms of small perturbations $\varepsilon_{m}$ and $\varepsilon_{n}$, the azimuthal angles of spins can be expressed as $\theta_{m}=\theta+\varepsilon_{m}$ and $\theta_{n}=\theta+\varepsilon_{n}$. After substituting these new angles in above equation number 1 , the cosine and sine terms can be expanded up to the second order of $\varepsilon_{m}$ and $\varepsilon_{n}$ as following.

\section{$E(\theta)=E_{0}+E(\varepsilon)+E\left(\varepsilon^{2}\right)$}

Here, $E_{0}=-\frac{1}{2} \sum_{m, n=1}^{N}\left(J Z_{|m-n|}-\frac{\omega}{4} \Phi_{|m-n|}\right)+\frac{3 \omega}{8} \cos 2 \theta \sum_{m, n=1}^{N} \Phi_{|m-n|}$

$$
-\cos ^{2} \theta \sum_{m=1}^{N} D_{m}^{(2)}-\cos ^{4} \theta \sum_{m=1}^{N} D_{m}^{(4)}-N\left(H_{\text {in }} \sin \theta+H_{\text {out }} \cos \theta-\frac{N_{d}}{\mu_{0}}+K_{s} \sin 2 \theta\right)
$$

$$
E(\varepsilon)=-\frac{3 \omega}{8} \sin 2 \theta \sum_{m, n=1}^{N} \Phi_{|m-n|}\left(\varepsilon_{m}+\varepsilon_{n}\right)+\sin 2 \theta \sum_{m=1}^{N} D_{m}^{(2)} \varepsilon_{m}+2 \cos ^{2} \theta \sin 2 \theta \sum_{m=1}^{N} D_{m}^{(4)} \varepsilon_{m}
$$




$$
\begin{aligned}
& -H_{\text {in }} \cos \theta \sum_{m=1}^{N} \varepsilon_{m}+H_{\text {out }} \sin \theta \sum_{m=1}^{N} \varepsilon_{m}-2 K_{s} \cos 2 \theta \sum_{m=1}^{N} \varepsilon_{m} \\
E\left(\varepsilon^{2}\right)= & \frac{1}{4} \sum_{m, n=1}^{N}\left(J Z_{|m-n|}-\frac{\omega}{4} \Phi_{|m-n|}\right)\left(\varepsilon_{m}-\varepsilon_{n}\right)^{2}-\frac{3 \omega}{16} \cos 2 \theta \sum_{m, n=1}^{N} \Phi_{|m-n|}\left(\varepsilon_{m}+\varepsilon_{n}\right)^{2} \\
& -\left(\sin ^{2} \theta-\cos ^{2} \theta\right) \sum_{m=1}^{N} D_{m}{ }^{(2)} \varepsilon_{m}{ }^{2}+2 \cos ^{2} \theta\left(\cos ^{2} \theta-3 \sin ^{2} \theta\right) \sum_{m=1}^{N} D_{m}{ }^{(4)} \varepsilon_{m}{ }^{2} \\
& +\frac{H_{\text {in }}}{2} \sin \theta \sum_{m=1}^{N}{\varepsilon_{m}}^{2}+\frac{H_{o u t}}{2} \cos \theta \sum_{m=1}^{N} \varepsilon_{m}{ }^{2}-\frac{N_{d}}{2 \mu_{0}} \sum_{m, n=1}^{N}\left(\varepsilon_{m}-\varepsilon_{n}\right)^{2} \\
& +2 K_{s} \sin 2 \theta \sum_{m=1}^{N} \varepsilon_{m}{ }^{2}
\end{aligned}
$$

After using the constraint $\sum_{m=1}^{N} \varepsilon_{m}=0, E(\varepsilon)=\vec{\alpha} \cdot \vec{\varepsilon}$

Here, $\vec{\alpha}(\varepsilon)=\vec{B}(\theta) \sin 2 \theta$ are the terms of matrices with

$$
B_{\lambda}(\theta)=-\frac{3 \omega}{4} \sum_{m=1}^{N} \Phi_{|\lambda-m|}+D_{\lambda}^{(2)}+2 D_{\lambda}^{(4)} \cos ^{2} \theta
$$

Also, $E\left(\varepsilon^{2}\right)=\frac{1}{2} \vec{\varepsilon} \cdot C \cdot \vec{\varepsilon}$

Here, the elements of matrix are given by,

$$
\begin{aligned}
C_{m n}= & -\left(J Z_{|m-n|}-\frac{\omega}{4} \Phi_{|m-n|}\right)-\frac{3 \omega}{4} \cos 2 \theta \Phi_{|m-n|}+\frac{2 N_{d}}{\mu_{0}} \\
& +\delta_{m n}\left\{\sum_{\lambda=1}^{N}\left[J Z_{|m-\lambda|}-\Phi_{|m-\lambda|}\left(\frac{\omega}{4}+\frac{3 \omega}{4} \cos 2 \theta\right)\right]-2\left(\sin ^{2} \theta-\cos ^{2} \theta\right) D_{m}{ }^{(2)}\right. \\
& \left.+4 \cos ^{2} \theta\left(\cos ^{2} \theta-3 \sin ^{2} \theta\right) D_{m}^{(4)}+H_{\text {in }} \sin \theta+H_{\text {out }} \cos \theta-\frac{4 N_{d}}{\mu_{0}}+4 K_{s} \sin 2 \theta\right\}
\end{aligned}
$$

Therefore, the total magnetic energy given in equation (2) can be reduced to

$E(\theta)=E_{0}+\vec{\alpha} \cdot \vec{\varepsilon}+\frac{1}{2} \vec{\varepsilon} \cdot C \cdot \vec{\varepsilon}=\mathrm{E}_{0}-\frac{1}{2} \vec{\alpha} \cdot C^{+} \cdot \vec{\alpha}$

Here $C^{+}$is the pseudo-inverse given by

$$
C . C^{+}=1-\frac{E}{N} \text {. }
$$

Here $E$ is the matrix with all elements $E_{m n}=1$. In this 2-D classical model, spin $(S)$ continuously varies from 0 to 1 contrary to quantum model where spin is quantized. The magnetic energy in Joules per unit spin is given throughout this manuscript. 


\section{RESULTS AND DISCUSSION}

Figure 1 shows the graph of $\frac{E(\theta)}{\omega}$ versus angle of bcc structured ferromagnetic thin films for $\frac{J}{\omega}=\frac{D_{m}{ }^{(4)}}{\omega}=\frac{K_{s}}{\omega}=\frac{N_{d}}{\mu_{o} \omega}=\frac{H_{\text {in }}}{\omega}=\frac{H_{\text {out }}}{\omega}=\frac{D_{m}{ }^{(2)}}{\omega} 10$ and 5 spin layers $(\mathrm{N}=5)$. For bcc (001) structure, $\mathrm{Z}_{0}=0, \mathrm{Z}_{1}=4, \mathrm{Z}_{2}=0 \quad$ and $\Phi_{0}=5.8675, \Phi_{1}=2.7126$ (Hucht and Usadel, 1997; Hucht and Usadel, 1999; Usadel and Hucht, 2002). Maximum and minimum of this graph correspond to hard and easy direction of Magnetization. Easy and hard directions are $30.91107^{\circ}$ and $145.4568^{\circ}$, respectively. The energies in easy and hard directions are 243.2086 and -64.6387 , respectively. Similarly easy and hard directions were determined for other $\mathrm{N}$ values. The corresponding easy and hard directions and energies in easy and hard directions for bcc structured ferromagnetic films from $\mathrm{N}=5$ to 20 spin layers are tabulated in table 1.

Figure 2 shows the graph of angle of easy direction measured with normal drawn to the film plane versus number of spin layers. According to this graph, the easy axis gradually rotates from out of plane to in plane direction of the film with the increase of number spin layers by indicating a preferred in plane orientation of easy axis at higher thicknesses. However, the angle increases in a step like pattern according to the graph. According to the column six in table 1 , the energy required to rotate spins from easy to hard direction gradually increases with the number of spin layers. The angle between easy and hard directions varies between $101^{\circ}$ and $116^{\circ}$ approximately in an unsystematic matter.

Figure 3 shows the graph of $\frac{E(\theta)}{\omega}$ versus angle of fcc structured ferromagnetic thin films for

$\frac{J}{\omega}=\frac{D_{m}{ }^{(4)}}{\omega}=\frac{K_{s}}{\omega}=\frac{N_{d}}{\mu_{o} \omega}=\frac{H_{\text {in }}}{\omega}=\frac{H_{\text {out }}}{\omega}=\frac{D_{m}{ }^{(2)}}{\omega} 10$ and 5 spin layers $(\mathrm{N}=5)$. For fcc(001) structure, $\mathrm{Z}_{0}=4, \mathrm{Z}_{1}=4, \mathrm{Z}_{2}=0$ and $\Phi_{0}=9.0336, \Phi_{1}=1.4294$ (Hucht and Usadel, 1997; Hucht and Usadel, 1999; Usadel and Hucht, 2002). Easy and hard directions are $32.72735^{\circ}$ and $140.0022^{\circ}$, respectively. The energies in easy and hard directions are 243.2285 and -65.2182 , respectively. Similarly easy and hard directions were determined for other $\mathrm{N}$ values. The corresponding easy and hard directions and energies in easy and hard directions for fcc structured ferromagnetic films from 5 to 20 spin layers are given in table 2 . The angle between easy and hard directions varies between $101^{\circ}$ and $112^{\circ}$ approximately in an unsystematic matter. Similarly, the hard axis rotates toward negative direction of the normal drawn to the film plane with the increase of number of spin layers to keep above angle between easy and hard directions.

Figure 4 indicates the graph of angle of easy direction measured with normal drawn to the film plane versus number of spin layers. The easy axis gradually rotates from out of plane to in plane direction of the film with the increase of number spin layers by indicating a preferred in plane orientation of easy axis at higher thicknesses for fcc structure too. According to the table 2, the energy required to rotate spins from easy to hard direction gradually increases with the number of spin layers for fcc structure too.

Easy axis orientation of magnetic thin films depends on deposition temeperature, annealing conditions, orientation of substarte, sputtering gas pressure, type of sputtering gas, deposition rate and the thickness of the film (Samarasekara, 2002). Previuosly, the orientation of easy axis of ferromagnetic and ferrite thin films has been theoreticall explained using Heisenberg Hamiltonian (Samarasekara and Gunawardhane, 2011; Samarasekara and Saparamadu, 2012; Samarasekara and Saparamadu, 2013). Magnetic energy due to spin exchange interaction, second order magnetic anisotropy, fourth order magnetic anisotropy, magnetic field, stress induced anisotropy decreases with the number of layers (Samarasekara, 2010). Due to the interaction between two spins, the spin exchange interaction and magnetic dipole interaction originate. Origin of magnetic anisotropy is the coupling between orbital angular momentum and spin angular moment of the atom. Due to the stress arising in the heating or cooling process of the thin film, the stress induced anisotropy takes place (Samarasekara, 2002). 
Table 1: Magnetic easy and hard directions with number of spin layers for bcc structure.

\begin{tabular}{|c|c|c|c|c|c|c|}
\hline $\begin{array}{c}\mathrm{N} \\
\text { (Number } \\
\text { of spin } \\
\text { layers) }\end{array}$ & $\begin{array}{c}\theta \text { (easy) } \\
\text { In degrees }\end{array}$ & 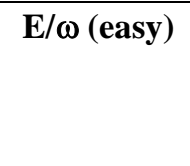 & $\begin{array}{c}\theta \text { (hard) } \\
\text { In degrees }\end{array}$ & E/w (hard) & $\begin{array}{c}\Delta \mathrm{E}= \\
\mathrm{E}(\text { easy)- } \\
\mathbf{E}(\mathbf{h a r d})\end{array}$ & $\begin{array}{c}\Delta \theta=\theta(\text { hard }) \\
-\theta(\text { easy }) \\
\text { In degrees }\end{array}$ \\
\hline 5 & 30.91107 & -243.2086 & 145.4568 & -64.6387 & 178.5699 & 114.5457 \\
\hline 6 & 32.72735 & -292.9421 & 139.9965 & -79.9431 & 212.999 & 107.2692 \\
\hline 7 & 32.72735 & -342.3487 & 134.5477 & -94.4943 & 247.8544 & 101.8204 \\
\hline 8 & 34.54363 & -391.5523 & 138.1802 & -108.6179 & 282.9344 & 103.6366 \\
\hline 9 & 34.54363 & -440.5596 & 141.8185 & -122.0178 & 318.5418 & 107.2749 \\
\hline 10 & 36.36563 & -487.1121 & 147.2559 & -131.6012 & 355.5109 & 110.8903 \\
\hline 11 & 36.36563 & -538.5211 & 143.6405 & -143.5001 & 395.021 & 107.2749 \\
\hline 12 & 38.18191 & -586.9962 & 143.6348 & -158.3737 & 428.6225 & 105.4529 \\
\hline 13 & 38.18191 & -635.8215 & 141.8185 & -163.6276 & 472.1939 & 103.6366 \\
\hline 14 & 39.99818 & -684.0491 & 156.3659 & -179.2071 & 504.842 & 116.3677 \\
\hline 15 & 39.99818 & -732.0545 & 154.5439 & -184.7936 & 547.2609 & 114.5457 \\
\hline 16 & 41.82019 & -780.1839 & 143.6348 & -210.5787 & 569.6052 & 101.8146 \\
\hline 17 & 41.82019 & -829.9423 & 149.0893 & -222.1916 & 607.7507 & 107.2691 \\
\hline 18 & 43.63647 & -879.0021 & 150.9171 & -230.8011 & 648.201 & 107.2806 \\
\hline 19 & 43.63647 & -927.9395 & 149.0893 & -240.7005 & 687.239 & 105.4528 \\
\hline 20 & 45.45274 & -975.1917 & 150.9114 & -251.8372 & 723.3545 & 105.4587 \\
\hline
\end{tabular}

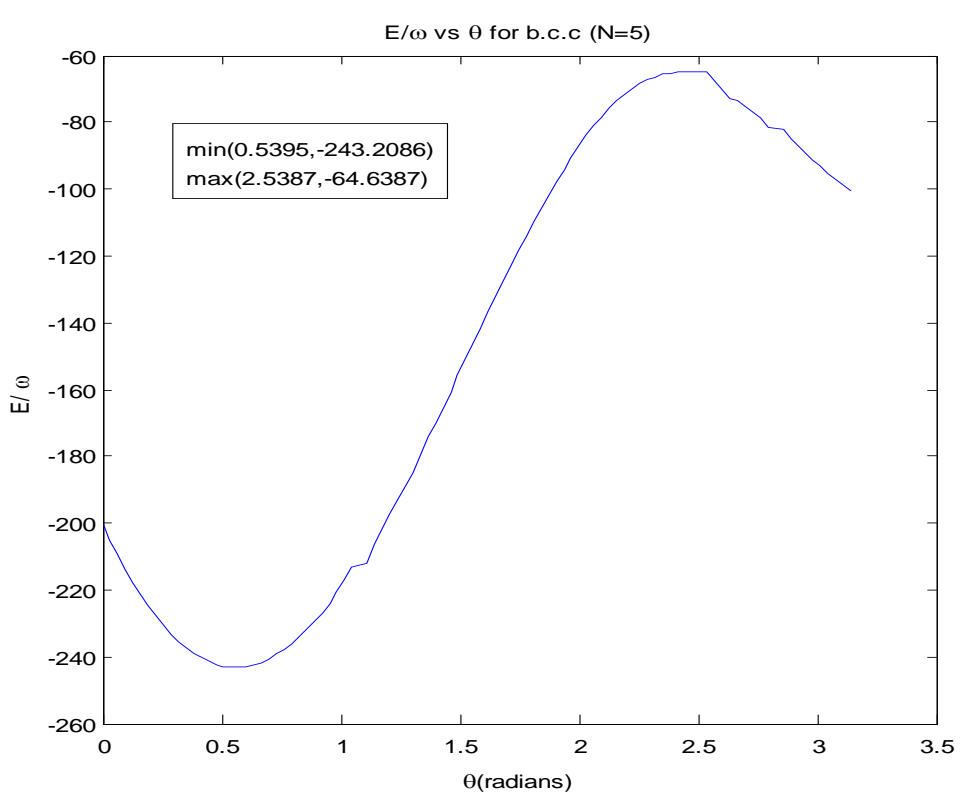

Figure 1: $\frac{E(\theta)}{\omega}$ versus angle for bcc structured film with five spin layers. 


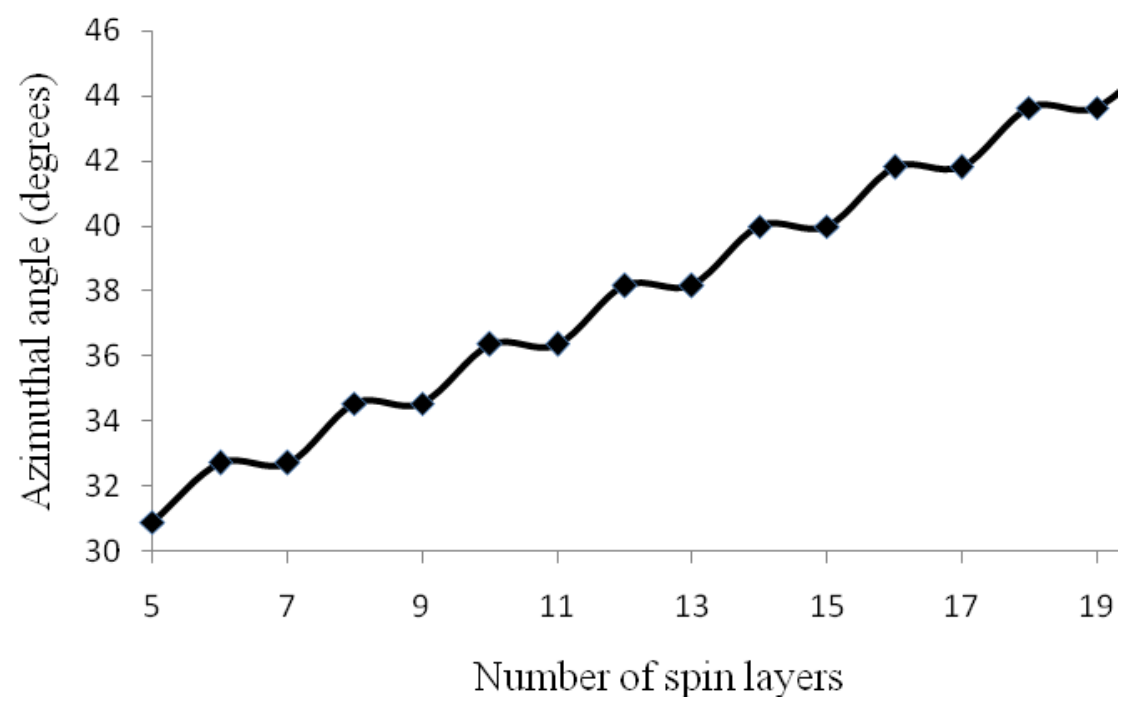

Figure 2: Graph of azimuthal angle of easy direction versus number of spin layers for bcc structured ferromagnetic films.

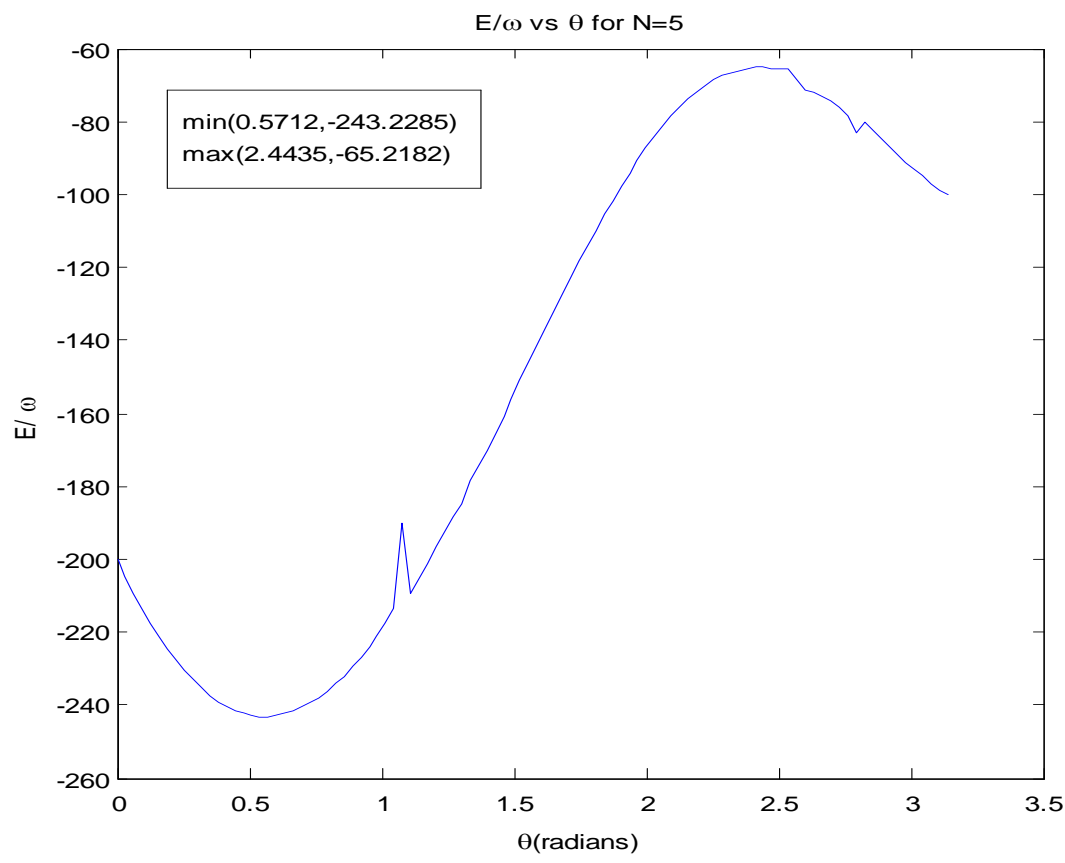

Figure 3: $\frac{E(\theta)}{\omega}$ versus angle for fcc structured film with five spin layers. 
Table 2: Variation of easy and hard directions with number of layers for fcc structure.

\begin{tabular}{|c|c|c|c|c|c|c|}
\hline $\begin{array}{c}\mathrm{N} \\
\text { (Number } \\
\text { of spin } \\
\text { layers) }\end{array}$ & $\begin{array}{c}\theta \text { (easy) } \\
\text { In degrees }\end{array}$ & 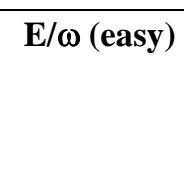 & $\begin{array}{c}\theta \text { (hard) } \\
\text { In degrees }\end{array}$ & E/ $\omega$ (hard) & $\begin{array}{c}\Delta \mathbf{E}=\mathbf{E}(\text { easy })- \\
\mathbf{E}(\text { hard })\end{array}$ & $\begin{array}{c}\Delta \theta=\theta(\text { hard }) \\
-\theta(\text { easy }) \\
\text { In degrees }\end{array}$ \\
\hline 5 & 32.72735 & -243.2285 & 140.0022 & -65.2182 & 178.0103 & 107.2749 \\
\hline 6 & 32.72735 & -292.9576 & 140.0022 & -80.1233 & 212.8343 & 107.2749 \\
\hline 7 & 32.72735 & -342.3769 & 134.5477 & -94.8201 & 247.5568 & 101.8204 \\
\hline 8 & 34.54363 & -391.5877 & 138.1802 & -109.1105 & 282.4772 & 103.6366 \\
\hline 9 & 34.54363 & -440.5967 & 141.8185 & -122.5244 & 318.0723 & 107.2749 \\
\hline 10 & 34.54363 & -488.2281 & 147.2559 & -137.2011 & 351.027 & 112.7123 \\
\hline 11 & 36.36563 & -538.3510 & 143.6462 & -147.9012 & 390.4498 & 107.2806 \\
\hline 12 & 38.18191 & -635.6171 & 143.6348 & -176.5205 & 459.0966 & 105.4529 \\
\hline 13 & 38.18191 & -635.6171 & 145.4568 & -169.7078 & 465.9093 & 107.2749 \\
\hline 14 & 39.99818 & -683.9634 & 147.2731 & -183.4255 & 500.5379 & 107.2749 \\
\hline 15 & 39.99818 & -732.2429 & 145.4568 & -193.2809 & 538.962 & 105.4586 \\
\hline 16 & 41.82019 & -780.5705 & 152.7276 & -206.6594 & 573.9111 & 110.9074 \\
\hline 17 & 41.82019 & -829.8509 & 147.2731 & -218.3991 & 611.4518 & 105.4529 \\
\hline 18 & 43.63647 & -878.7845 & 149.0893 & -233.5521 & 645.2324 & 105.4528 \\
\hline 19 & 43.63647 & -927.7699 & 150.9114 & -238.9065 & 688.8634 & 107.2749 \\
\hline 20 & 45.45274 & -975.9370 & 154.5439 & -243.5472 & 732.3898 & 109.0912 \\
\hline
\end{tabular}

The difference between the thermal expansion coefficients of the film material and the substrate is the reason for the stress induced anisotropy. However, magnetic energy due to magnetic dipole interaction and demagnetization factor increases with number of layers. Although the origin of spin exchange interaction and magnetic dipole interaction is the interaction between two spins, spin exchange interaction and magnetic dipole interaction vary in opposite ways with the increase of number of spin layers. The sign of spin exchange interaction and magnetic dipole interaction are negative and positive, respectively, in equation number 1 . The reason for in plane orientation of easy axis is attributed to the domination of energy due to magnetic dipole interaction and demagnetization factor at higher thicknesses. Although the demagnetization factor in the direction perpendicular to the film plane slightly decreases with the thickness, the magnetic energy due to demagnetization factor increases with the thickness according to our equations. 


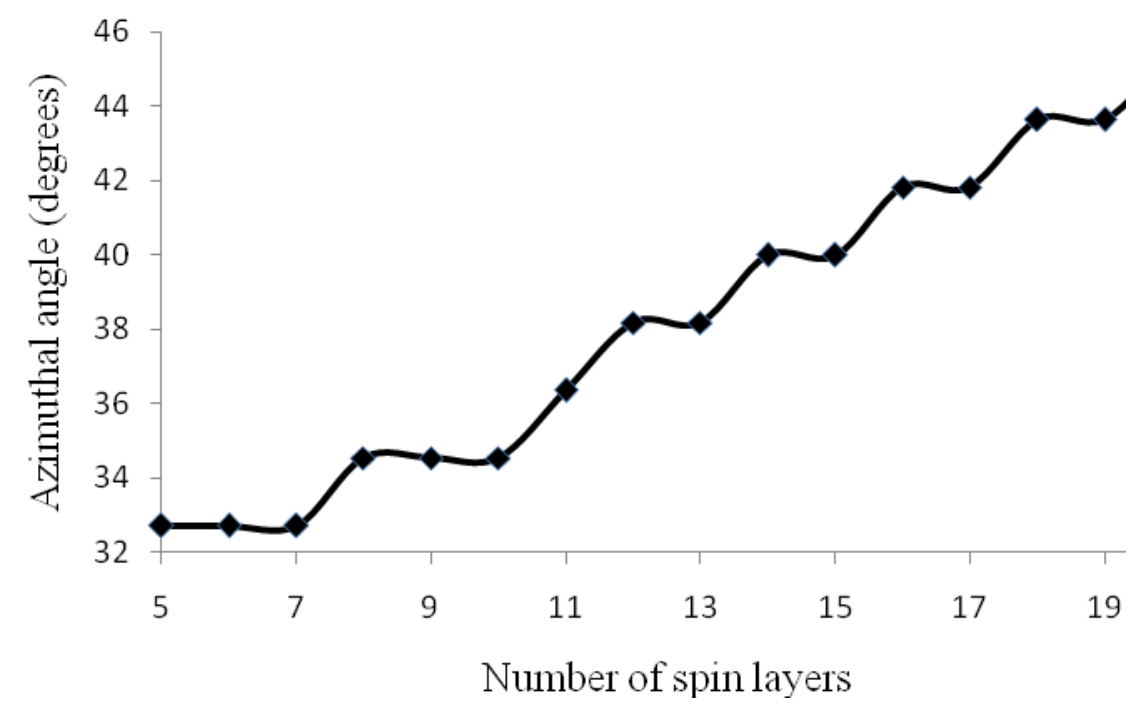

Figure 4: Azimuthal angle of easy direction versus number of spin layers for fcc structured ferromagnetic films.

According to some experimental data, the easy axis of ferromagnetic thin films rotates from out of plane to in plane direction with the increase of the thickness. The magnetic easy axis of sputtered ferromagnetic Ni films indicates a preferred in plane orientation at higher thicknesses (Parlak et al., 2015). According to this experimental data, the spin reorientation transition occurs from in plane to out plane at film thickness in between 14 and $24{ }^{0} \mathrm{~A}$. The magnetic easy axis of ferromagnetic $\mathrm{Fe}$ thin films fabricated by electron beam evaporation indicates a preferred in plane orientation above thicknesses of 2 monolayers (Araya-Pochet et $a l ., 1988)$. If number of spins per layer is $n$, then the total number of spins in the film with $N$ number of spin layers is $n N$. So the number of spins in a ferromagnetic film increases with the number of layers. As a matter of fact, the energy $(\Delta \mathrm{E})$ required to rotate spins from easy to hard (or vice versa) direction gradually increases with the number of spin layers. The films with higher or smaller $\Delta \mathrm{E}$ values are useful in the applications where hard or soft magnetic materials are required, respectively. Soft or hard magnetic materials are used in rewritable or permanent magnetic memory devices, respectively.

\section{CONCLUSION}

The variation of magnetic easy and hard directions and the energy required to rotate from easy to hard direction were investigated for the bcc and fcc structured films with five to twenty spin layers. The magnetic easy axis gradually rotates from the out of plane to in plane direction with the increase of number of spin layers. Because the energy due to magnetic dipole interaction and demagnetization factor dominates the energy due to magnetic anisotropies at higher thicknesses, the film indicates a preferred in plane orientation at higher thicknesses. In addition, the energy required to rotate all the spins from easy to hard (or vice versa) direction gradually increases with the number of spin layers. Because the total number of spins increases with the number of spin layers, the energy required to rotate all the spins increases with the number of layers. However, the angles between easy and hard directions don't vary in a systematic manner for both fcc and bcc structured ferromagnetic films.

\section{REFERENCES}

Araya-Pochet J, Ballentine C.A and Erskine J.L. (1988). Thickness and temperature dependent spin anisotropy of ultrathin epitaxial Fe films on Ag(100). Physical Review B 38(11): 7846- 7849

Cates James C. and Alexander Jr Chester. (1994). Theoretical study of magnetostriction in FeTaN thin films. Journal of Applied Physics 75: 67546756.

Dantziger M, Glinsmann B, Scheffler S, Zimmermann $B$ and Jensen P.J. (2002). In-plane dipole coupling anisotropy of a square ferromagnetic Heisenberg monolayer. Physical Review B 66: 094416.

Ernst A, Lueders M, Temmerman W.M, Szotek Z and Van der Laan G. (2000). Theoretical study of magnetic layers of nickel on copper; dead or 
live?. Journal of Physics: Condensed matter 12(26): 5599-5606.

Hucht A and Usadel K.D. (1997). Reorientation transition of ultrathin ferromagnetic films. Physical Review B 55: 12309.

Hucht A and Usadel K.D. (1999). Theory of the spin reorientation transition of ultra-thin ferromagnetic films. Journal of Magnetism and Magnetic materials 203(1): 88-90.

Kovachev St and Wesselinowa J.M. (2009). Theoretical study of multiferroic thin films based on a microscopic model. Journal of Physics: Condensed matter 21(22): 225007.

Nowak U. (1995). Magnetisation reversal and domain structure in thin magnetic films: Theory and computer simulation. IEEE transaction on magnetics 31(6-2): 4169-4171.

Parlak U, Akoz M.E, Tokdemir Ozturk S and Erkovan M. (2015). Thickness dependent magnetic properties of polycrystalline nickel thin films. Acta Physica Polonica A 127(4): 995-997.

Radomska Anna and Balcerzak Tadeusz (2003). Theoretical studies of model thin EuTe films with surface elastic stresses. Central European Journal of Physics 1(1): 100-117.

Samarasekara P. (2002). Easy Axis Oriented Lithium Mixed Ferrite Films Deposited by the PLD Method. Chinese Journal of Physics 40(6): 631636.

Samarasekara P. (2008). Influence of third order perturbation on Heisenberg Hamiltonian of thick ferromagnetic films. Electronic Journal of Theoretical Physics 5(17): 227-236.

Samarasekara P., Abeyratne M.K. and Dehipawalage S. (2009). Heisenberg Hamiltonian with Second Order Perturbation for Spinel Ferrite Thin Films. Electronic Journal of Theoretical Physics 6(20): 345-356.

Samarasekara P and William A. Mendoza (2010). Effect of third order perturbation on Heisenberg Hamiltonian for non-oriented ultra-thin ferromagnetic films. Electronic Journal of Theoretical Physics 7(24): 197-210.

Samarasekara P. (2010). Determination of energy of thick spinel ferrite films using Heisenberg
Hamiltonian with second order perturbation. Georgian electronic scientific journals: Physics 1(3): 46-49.

Samarasekara P. (2011). Investigation of Third Order Perturbed Heisenberg Hamiltonian of Thick Spinel Ferrite Films. Inventi Rapid: Algorithm Journal 2(1): 1-3.

Samarasekara P and William A. Mendoza. (2011). Third Order Perturbed Heisenberg Hamiltonian of Spinel Ferrite Ultra-thin films. Georgian electronic scientific journals: Physics 1(5): 1524.

Samarasekara P and Gunawardhane N.H.P.M. (2011). Explanation of easy axis orientation of ferromagnetic films using Heisenberg Hamiltonian. Georgian electronic scientific journals: Physics 2(6): 62-69.

Samarasekara P and Saparamadu Udara (2012). Investigation of Spin Reorientation in Nickel Ferrite Films. Georgian electronic scientific journals: Physics 1(7): 15-20.

Samarasekara P and Saparamadu Udara (2013). Easy axis orientation of barium hexa-ferrire films as explained by spin reorientation. Georgian electronic scientific journals: Physics 1(9): 1015.

Spisak D and Hafner J. (2005). Theoretical study of $\mathrm{FeCo} / \mathrm{W}(110)$ surface alloys. Journal of Magnetism and Magnetic Materials 286: 386389.

Tsai Shan-Ho, Landau D.P and Schulthess Thomas C. (2003). Effect of interfacial coupling on the magnetic ordering in ferro-antiferromagntic bilayers. Journal of Applied Physics 93(10): 8612-8614.

Usadel K.D and Hucht A. (2002). Anisotropy of ultrathin ferromagnetic films and the spin reorientation transition. Physical Review B 66: 024419.

Zhao D, Feng Liu, Huber D.L and Lagally M.G. (2002). Step-induced magnetic-hysteresis anisotropy in ferromagnetic thin films. Journal of Applied Physics 91(5): 3150-3153. 
